\title{
Mesoscale theory of grains and cells: crystal plasticity and coarsening
}

\author{
Surachate Limkumnerd* and James P. Sethnd \\ Laboratory of Atomic and Solid State Physics, Clark Hall, \\ Cornell University, Ithaca, NY 14853-2501, USA
}

(Dated: September 20, 2018)

\begin{abstract}
Solids with spatial variations in the crystalline axes naturally evolve into cells or grains separated by sharp walls. Such variations are mathematically described using the Nye dislocation density tensor. At high temperatures, polycrystalline grains form from the melt and coarsen with time: the dislocations can both climb and glide. At low temperatures under shear the dislocations (which allow only glide) form into cell structures. While both the microscopic laws of dislocation motion and the macroscopic laws of coarsening and plastic deformation are well studied, we hitherto have had no simple, continuum explanation for the evolution of dislocations into sharp walls. We present here a mesoscale theory of dislocation motion. It provides a quantitative description of deformation and rotation, grounded in a microscopic order parameter field exhibiting the topologically conserved quantities. The topological current of the Nye dislocation density tensor is derived from a microscopic theory of glide driven by Peach-Koehler forces between dislocations using a simple closure approximation. The resulting theory is shown to form sharp dislocation walls in finite time, both with and without dislocation climb.
\end{abstract}

PACS numbers: $61.72 . \mathrm{Bb}, 61.72 . \mathrm{Lk}, 62.20 \mathrm{Fe}$

Crystals, when formed or deformed, relax by developing walls. Common metals (coins, silverware) are polycrystalline: the atoms locally arrange into grains each with a specific crystalline lattice orientation, separated by sharp, flat walls called grain boundaries. When metals are deformed (pounded or permanently bent) new cell walls form inside each grain ${ }^{1,2.3}$. Until now, our only convincing understanding of why crystals form walls has been detailed and microscopic. Our new theory provides an elegant, continuum description of cell wall formation as the development of a shock front - a phenomenon hitherto associated with traffic jams and sonic booms.

Continuum theories of dislocation dynamics are not new ${ }^{4.5 .6 .7}$. Various approaches have been used to study dislocation pattern formation. Simplified diffusion models have been used to describe persistent slip bands ${ }^{8}$, dislocation cell structures during multiple slip $\underline{\underline{9}}$, and dislocation vein structures 10 . Models similar in spirit to ours have recently been analyzed both for single-slip 11.12 .13 and in three dimensions 14 . None of these approaches have yielded the sharp, wall-like singularities characteristic of grain boundaries and deformation-induced cell structures (see, however ${ }^{15}$ ).

Continuum plasticity theory, as used in practical engineering codes, describes the deformation gradient $\left(\partial_{i} u_{j}\right.$, for displacement field $\mathbf{u}$ ) as the sum $\partial_{i} u_{j}=\beta_{i j}^{\mathrm{E}}+\beta_{i j}^{\mathrm{P}}$ of an elastic, reversible distortion $\boldsymbol{\beta}^{\mathrm{E}}$ and a permanent plastic distortion $\boldsymbol{\beta}^{\mathrm{P}}$. The plastic distortion involves the creation of microscopic dislocations, and cannot be written as a gradient of a single-valued displacement field. Integrating around a loop $L$ enclosing a surface $S$, the change in such a hypothetical plastic distortion field $\Delta \mathbf{u}^{\mathrm{P}}$ can be written using Stokes theorem as

$$
\Delta u_{j}^{\mathrm{P}}=-\oint_{L} \beta_{i j}^{\mathrm{P}} d x_{i}=-\int_{S} \varepsilon_{i l m} \frac{\partial \beta_{m j}^{\mathrm{P}}}{\partial x_{l}} d S_{i}=\int_{S} \rho_{i j} d S_{i}
$$

where the Nye dislocation density tensor

$$
\rho_{i j}(\mathbf{x})=-\varepsilon_{i l m} \frac{\partial \beta_{m j}^{\mathrm{P}}}{\partial x_{l}}=\sum_{\alpha} t_{i}^{\alpha} b_{j}^{\alpha} \delta\left(\xi^{\alpha}\right)
$$

measures the net flux of dislocations $\alpha$, tangent to $\mathbf{t}$, with Burgers vector $\mathbf{b}$, in the (coarse-grained) neighborhood of $\mathbf{x}$. The microscopic statement that dislocations cannot end implies $\partial_{i} \rho_{i j}=0$, so the time evolution must be given in terms of a current ${ }^{6.7 .16} \boldsymbol{J}: \partial \rho_{i j} / \partial t=-\varepsilon_{i l m} \partial J_{m j} / \partial x_{l}$. From equation (2) we see that ${ }^{17}$

$$
J_{i j}=\partial \beta_{i j}^{\mathrm{P}} / \partial t .
$$

Thus the natural physicist's order parameter (the topologically conserved dislocation density $\boldsymbol{\rho}$ ) is a curl of the common engineering state variable (the plastic distortion field $\boldsymbol{\beta}^{\mathrm{P}}$ ). The focus of our manuscript will be the derivation of an evolution law (3) appropriate for scales large compared to the atoms but small compared to the cell structures and grain boundaries.

Notice that we only consider the net density of dislocations. We ignore the geometrically unneccessary dislocations (those with opposing Burgers vectors which cancel out in the net dislocation density) because they do not affect the long range strain fields or the misorientations at grain boundaries and cell walls. Macroscopically they are known to dominate dislocation entanglement and work hardening, and are included in previous continuum theories of plasticity $\frac{8.11,12,13.18 .19}{}$. Much of the macroscopic cancellation in net dislocation density comes from the near alternation of the net rotations in the series of cell walls ${ }^{20}$. Our focus on the sub-cellular, subgrain length scales and our current omission of dislocation tangling make keeping only the net dislocation density natural for our purposes. We also do not explicitly incorporate a yield surface, because we hope eventually to explain work 
hardening and yield surfaces as properties which emerge from the intermediate length-scale theory.

We motivate our evolution law from the microscopic Peach-Koehler force on a section of a dislocation line due to the stress field $\boldsymbol{\sigma}$ present at that point: $f_{i}^{\mathrm{PK}}=$ $-\varepsilon_{i j k} t_{j} b_{l} \sigma_{k l}$. The current $\boldsymbol{J}$ of a single dislocation moving with velocity $\mathbf{v}$ is $J_{i j}=\varepsilon_{i l m} t_{l} b_{j} v_{m} \delta(\xi)$. If we treat dislocation glide and climb on an equal footing (a crude model for metals at high temperatures), the dislocation will move in the direction of the applied force $\mathbf{v} \propto \mathbf{f}^{\mathrm{PK}}$. For a single dislocation, tuning the climb component with a parameter $\lambda$, we find

$$
\begin{aligned}
J_{i j}=D[ & \varepsilon_{i l m} t_{l} b_{j} \varepsilon_{m p q} \sigma_{p r} t_{q} b_{r} \delta(\xi) \\
& \left.-\frac{\lambda}{3} \delta_{i j} \varepsilon_{k l m} t_{l} b_{k} \varepsilon_{m p q} \sigma_{p r} t_{q} b_{r} \delta(\xi)\right] .
\end{aligned}
$$

Here $D$ is a materials constant with units of [length $]^{2}[$ time $] /[$ mass $]$ giving the mobility of dislocation glide. At $\lambda=0$ climb and glide have equal mobilities, and at $\lambda=1 \boldsymbol{J}$ is traceless and hence only glide is allowed. Coarse graining over many dislocations,

$$
J_{i j}=D_{i j k m p q r s} \sigma_{p q} \rho_{k m r s}^{(4)}
$$

where $D_{i j k m p q r s}=D / 2\left[\delta_{i q} \delta_{j m} \delta_{k r} \delta_{p s}-\delta_{i r} \delta_{j m} \delta_{k q} \delta_{p s}-\right.$ $\left.\lambda / 3\left(\delta_{i j} \delta_{m q} \delta_{k r} \delta_{p s}-\delta_{i j} \delta_{m r} \delta_{k q} \delta_{p s}\right)\right]$ is an eighth rank isotropic tensor specific to Peach-Koehler model, and $\rho_{k m r s}^{(4)}$ is a higher order Nye dislocation tensor. Thus that the evolution of $\boldsymbol{\rho}$ depends upon the new object $\boldsymbol{\rho}^{(4)}$.

We now need to perform a closure approximation, writing $\boldsymbol{\rho}^{(4)}$ in terms of $\boldsymbol{\rho}$ (as in Hartree-Fock and in theories of turbulence). The only choice $\boldsymbol{\rho}^{(4)} \rightarrow \boldsymbol{\rho} \otimes \boldsymbol{\rho}$ that guarantees a decrease of elastic energy with time ${ }^{21}$ is

$$
\begin{aligned}
\rho_{i j k m}^{(4)} & =\sum_{\alpha} t_{i}^{\alpha} b_{j}^{\alpha} t_{k}^{\alpha} b_{m}^{\alpha} \delta\left(\xi^{\alpha}\right) \\
& \simeq C\left[\sum_{\alpha} t_{i}^{\alpha} b_{j}^{\alpha} \delta\left(\xi^{\alpha}\right)\right]\left[\sum_{\alpha^{\prime}} t_{k}^{\alpha^{\prime}} b_{m}^{\alpha^{\prime}} \delta\left(\xi^{\alpha^{\prime}}\right)\right]=C \rho_{i j} \rho_{k m}
\end{aligned}
$$

where $C$ has units of distance. The resulting evolution law for the plastic distortion tensor is

$$
\begin{aligned}
& \frac{\partial \beta_{i j}^{\mathrm{P}}}{\partial t}=\frac{C D}{2}\left[\left(\sigma_{i c} \rho_{a c}-\sigma_{a c} \rho_{i c}\right) \rho_{a j}\right. \\
& \left.-\frac{\lambda}{3} \delta_{i j}\left(\sigma_{k c} \rho_{a c}-\sigma_{a c} \rho_{k c}\right) \rho_{a k}\right]
\end{aligned}
$$

where $\boldsymbol{\rho}$ is a curl of $\boldsymbol{\beta}^{\mathrm{P}}$ (equation 2), and the stress $\boldsymbol{\sigma}$ (due to the long-range fields of the other dislocations) is written in Fourier space as 22

$$
\begin{aligned}
\tilde{\sigma}_{i j}(\mathbf{k})=K_{i j k l}(\mathbf{k}) \widetilde{\rho}_{k l}(\mathbf{k}), & \\
K_{i j k l}(\mathbf{k})=-\frac{i \mu k_{m}}{k^{2}}\left[\varepsilon_{i l m} \delta_{j k}\right. & +\varepsilon_{j l m} \delta_{i k} \\
& \left.+\frac{2 \varepsilon_{k l m}}{1-\nu}\left(\frac{k_{i} k_{j}}{k^{2}}-\delta_{i j}\right)\right] .
\end{aligned}
$$

For simplicity, we present solutions to our threedimensional theory for the special case of systems where the dislocation density varies only along one and two dimensions. In one dimension with variations only along $\hat{\mathbf{z}}$, our theory becomes local, with stress

$\boldsymbol{\sigma}(z)=-\mu\left(\begin{array}{ccc}\frac{2}{1-\nu}\left(\beta_{x x}^{\mathrm{P}}+\nu \beta_{y y}^{\mathrm{P}}\right) & \beta_{x y}^{\mathrm{P}}+\beta_{y x}^{\mathrm{P}} & 0 \\ \beta_{x y}^{\mathrm{P}}+\beta_{y x}^{\mathrm{P}} & \frac{2}{1-\nu}\left(\nu \beta_{x x}^{\mathrm{P}}+\beta_{y y}^{\mathrm{P}}\right) & 0 \\ 0 & 0 & 0\end{array}\right)$,

Nye dislocation tensor

$$
\rho_{x j}=\partial_{z} \beta_{y j}^{\mathrm{P}}, \quad \rho_{y j}=-\partial_{z} \beta_{x j}^{\mathrm{P}}, \quad \rho_{z j}=0,
$$

and evolution law

$$
\frac{\partial \boldsymbol{\beta}^{\mathrm{P}}}{\partial t}=-F\left(\boldsymbol{\beta}^{\mathrm{P}}\right) \frac{\partial \boldsymbol{\beta}^{\mathrm{P}}}{\partial z}
$$

with

$$
\begin{aligned}
F\left(\boldsymbol{\beta}^{\mathrm{P}}\right) & =-\frac{\partial w\left(\boldsymbol{\beta}^{\mathrm{P}}\right)}{\partial z}=-C D \mu \frac{\partial}{\partial z}\left[\frac{1}{2}\left(\beta_{x y}^{\mathrm{P}}+\beta_{y x}^{\mathrm{P}}\right)^{2}\right. \\
& \left.+\left(\beta_{x x}^{\mathrm{P}}{ }^{2}+\beta_{y y}^{\mathrm{P}}{ }^{2}\right)+\frac{\nu}{1-\nu}\left(\beta_{x x}^{\mathrm{P}}+\beta_{y y}^{\mathrm{P}}\right)^{2}\right] .
\end{aligned}
$$

Interestingly, $w\left(\boldsymbol{\beta}^{\mathrm{P}}\right)$ is the linear elastic energy density of the system, the effective force $F\left(\boldsymbol{\beta}^{\mathrm{P}}\right)$ is proportional to the $z$-component of the Peach-Koehler force, and the rate of change of the elastic energy density is simply $-F^{2}\left(\boldsymbol{\beta}^{\mathrm{P}}\right)$ (so the energy decreasing condition is explicit).

Our equation for $\boldsymbol{\beta}^{\mathrm{P}}$ in one dimension is hyperbolic: the method of characteristics may be applied, where the characteristics are the parameterized curves $(t(s), z(s))$ with $d t / d s=1, d z / d s=F\left(\boldsymbol{\beta}^{\mathrm{P}}\right)$. Along these curves,

$$
\frac{d \boldsymbol{\beta}^{\mathrm{P}}}{d s}=\frac{d t}{d s} \frac{\partial \boldsymbol{\beta}^{\mathrm{P}}}{\partial t}+\frac{d z}{d s} \frac{\partial \boldsymbol{\beta}^{\mathrm{P}}}{\partial z}=\frac{\partial \boldsymbol{\beta}^{\mathrm{P}}}{\partial t}+F\left(\boldsymbol{\beta}^{\mathrm{P}}\right) \frac{\partial \boldsymbol{\beta}^{\mathrm{P}}}{\partial z}=0,
$$

implying (from equation 11) that $\boldsymbol{\beta}^{\mathrm{P}}$ is constant along the characteristics. As with many hyperbolic systems, we observe shock formuation in finite time. Our analytic analysis of the shock asymptotics (in preparation) exhibits the jump in $\boldsymbol{\beta}^{\mathrm{P}}$, wall in $\boldsymbol{\rho}$, and stress jump described numerically below, with a $1 / \sqrt{t}$ time-dependence for the surrounding continuum $\boldsymbol{\beta}^{\mathrm{P}}$.

We simulate systems of spatial extent $L$ in one or two dimensions, with periodic boundary conditions and no external stress. The initial plastic distortion field $\boldsymbol{\beta}^{\mathrm{P}}$ is a Gaussian random field with mean square amplitude $\beta_{0}^{\mathrm{P}}$ and root-mean-square decay length approximately $L / 16$. In one dimension, we use the upwind scheme ${ }^{23}$ as implemented by Press et $a l^{24}$. In two dimensions, we calculate $\boldsymbol{\sigma}$ using Fourier methods and regularize our equations by adding a fourth-order numerical viscosity $-K \nabla^{4} \beta_{i j}^{\mathrm{P}}$ to equation 7 (A second-order viscosity $\nabla^{2} \beta_{i j}^{\mathrm{P}}$ was not as successful in suppressing the instabilities.) In one dimension, we have checked that the upwind scheme produces the same solution as the viscosity regularization. Unlike 
shock waves in fluids, where conservation laws tell us that the upwind/viscosity solution is the correct one, here the dynamics of the walls is not determined by the continuum theory. (We speculate that the wall dynamics regularization may be non-trivial and stochastic, emerging out of avalanches and critical depinning phenomena on sub-cell length scales - observed both experimentally 25 and theoretically ${ }^{26}$ in systems with only one active slip system.)

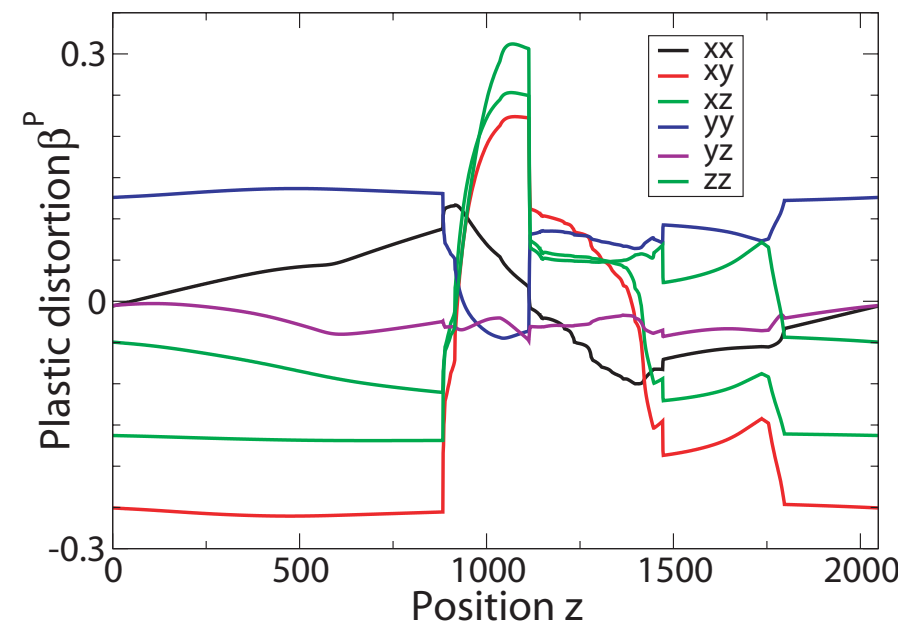

FIG. 1: Plastic distortion tensor component $\beta_{i j}^{\mathrm{P}}$ in one dimension allowing only glide motion, after time $t=20 L^{2} / D \mu$, with 2048 mesh points. The shocks or jumps in the values correspond to the cell walls. A stress-free wall (satisfying the Frank conditions) here would have no jump in $\beta_{x x}^{\mathrm{P}}, \beta_{y y}^{\mathrm{P}}$, or $\beta_{x y}^{\mathrm{P}}+\beta_{y x}^{\mathrm{P}}$.

Figure 1 shows the final state of the plastic distortion $\boldsymbol{\beta}^{\mathrm{P}}$ in a one-dimensional system evolving under dislocation glide only (corresponding to plastic deformation, which experimentally leads to cell structure formation). Jump singularities in $\boldsymbol{\beta}^{\mathrm{P}}$ form after a short time, representing a wall of dislocations and an abrupt change in lattice orientation. The cell walls in our model are not grain boundaries, because they do not correspond to pure, simple rotations (they do not satisfy the Frank conditions 27 ). There is a jump in the stress across each cell wall: the glide component of the forces from the two neighboring cells on $\rho$ in a wall is equal, opposite, and compressive. Our model therefore predicts that the initial formation of cell walls during low-temperature plastic deformation is driven by a stress jump due to non-cancellation of the stress field of the constituent dislocations. As our system evolves, new cell walls form (intriguingly similar to the cell refinement seen experimentally ${ }^{28,29}$ ) and the existing cell walls evolve to reduce their stress jumps.

Figure 2 shows the evolution of the plastic distortion in a system allowing both glide and climb, corresponding to high-temperature annealing of a plastically deformed structure. Here the initial singularities again are not grain boundaries, but walls of dislocations with net stress jumps. These walls then move and coalesce in response

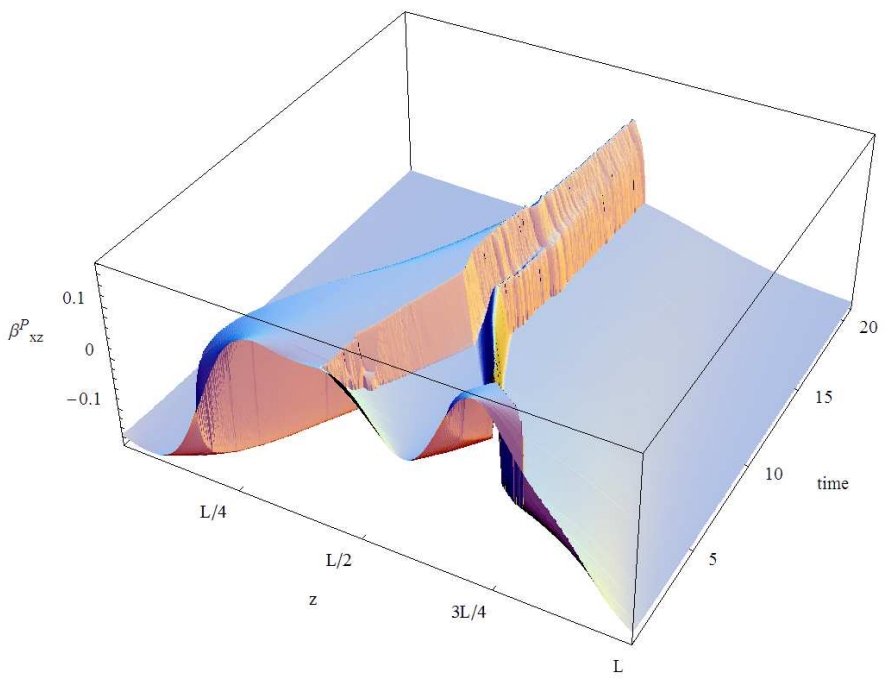

FIG. 2: The $x z$-component of the plastic distortion tensor in one dimension up to time $t=22 L^{2} / D \mu$, with 2048 mesh points. The evolution allows both glide and climb motions. The walls move and coalesce until only a single wall survives.

to the net force due to other dislocations, coarsening the resulting grain boundary structure.

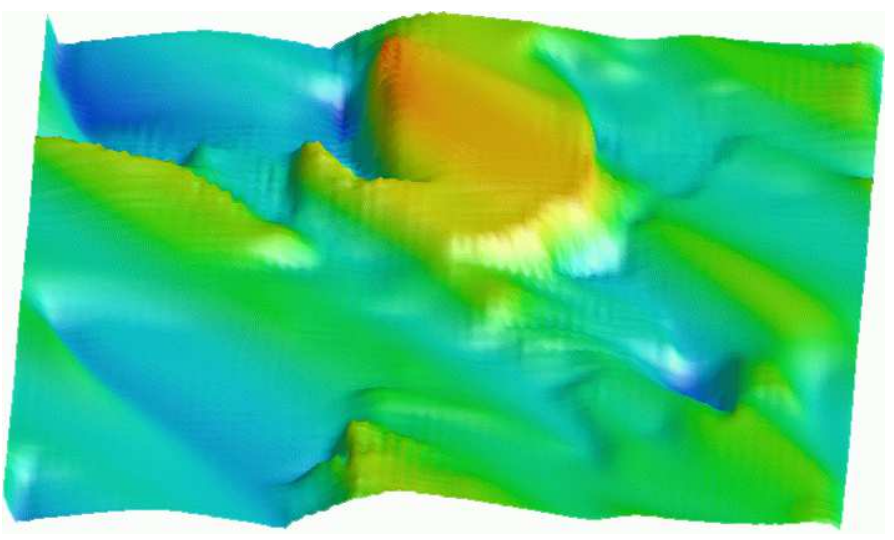

FIG. 3: The $y z$-component of the plastic distortion tensor allowing only glide, in two dimensions after time $t=$ $9.15 L^{2} / D \mu$.

Figure 3 shows the final state of $\boldsymbol{\beta}^{\mathrm{P}}$ in a twodimensional plasticity simulation (with glide and not climb). Notice the formation of sharp walls (mathematically demonstrated above only in one dimension). These walls separate relatively unstrained regions which we identify with the cells formed during plastic deformation. As in the one-dimensional simulations, the cell walls formed at short times have stress jumps.

Generically we expect our model to form sharp walls in finite time. Special initial conditions, however, may not form walls. Indeed, the case of a single slip system does not form singularities within our model-perhaps explaining why cell wall formation is not observed in 
Stage I hardening, or in dislocation-dynamics studies of single slip in two dimensions otherwise similar to our approach 11.12.13.

Our dislocation dynamics theory is minimalist: it ignores many features (geometrically unnecessary dislocations $^{11}$, slip systems ${ }^{30}$, dislocation tangling, yield surfaces, nucleation of new dislocations) that are known to be macroscopically important in real materials. It does incorporate cleanly and microscopically the topological constraints, long-range forces and energetics driving the dislocation dynamics. As hypothesized by the LEDS (low-energy dislocation structures) approach ${ }^{31.32}$, we find that a dynamics driven by minimizing energy (omitting tangling and nucleation) still produces cell boundary structures. Finally our theory, to our surprise, initially forms sharp walls that are not the usual zero-stress grain boundaries.

In condensed-matter physics, crystals are anomalous.
Most phases (liquid crystals, superfluids, superconductors, magnets) respond smoothly in space when strained. Crystals, when rotationally strained, form sharp walls separating cells or grains. Our analysis suggests that the formation of these walls can be understood as a shock formation in the dislocation dynamics.

\section{Acknowledgments}

We would like to thank Jean-Philippe Bouchaud, Valerie Coffman, Paul Dawson, Matthew Miller, Markus Rauscher, Wash Wawrzynek, and Steven $\mathrm{Xu}$ for helpful instruction on plasticity and numerical methods, and funding from NSF grants ITR/ASP ACI0085969 and DMR-0218475.
* Electronic address: sl129@cornell.edu

† URL: http://www. lassp. cornell.edu/sethna/sethna.html

1 D. Kuhlmann-Wilsdorf and N. Hansen, Scripta Metall. Mater. 25, 1557 (1991).

2 D. A. Hughes and N. Hansen, Metall. Trans. A 24, 2021 (1993).

3 D. A. Hughes and N. Hansen, Scripta Metall. Mater. 33, 315 (1995).

4 J. D. Eshelby, in Solid State Physics 3, edited by F. Seitz and D. Turnbull (Academic Press, San Diego, 1956), pp. 79-144.

${ }^{5}$ E. Kröner, Kontinuumstheorie der Versetzungen und Eigenspannungen (Springer-Verlag, Berlin, 1958).

6 A. M. Kosevich, Sov. Phys. JETP 15, 108 (1962).

7 T. Mura, Phil. Mag. 8, 843 (1963).

8 D. Walgraef and E. C. Aifantis, Int. J. Eng. Sci. 23, 1351 (1985).

9 P. Hähner, Appl. Phys. A 62, 473 (1996).

10 M. Saxlová, J. Kratochvil, and J. Zatloukal, Mater. Sci. Eng. A 234-236, 205 (1997).

11 M. Zaiser, M. C. Miguel, and I. Groma, Phys. Rev. B 64, 224102 (2001).

12 I. Groma, Phys. Rev. B 56, 5807 (1997), uses a similar approach to study plastic deformation in two dimensions with only one slip system (i.e., allowing only parallel edge dislocations with one direction of Burgers vector, leading to scalar order parameters). He does a closure-like factorization of a two-point dislocation density correlation function which leads to a theory with one fewer factor of $\rho$ in the evolution law. While we cannot generalize his approach to the three-dimensional tensor theory, we can reproduce his continuum theory by choosing $C(\boldsymbol{\rho})=C_{0} / \sqrt{\operatorname{tr}\left(\boldsymbol{\rho}^{\dagger} \boldsymbol{\rho}\right)}$ and specializing to two dimensions and one slip system.

13 B. Bakó and I. Groma, Phys. Rev. B 60, 122 (1999), explore 2D single-slip simulations of the earlier theory ${ }^{12}$, which do not form walls. We have verified that our model generates walls under multiple-slip even when we divide $C(\boldsymbol{\rho})$ by $\rho$ to reproduce their mode ${ }^{12}$.

14 A. El-Azab, Phys. Rev. B 61, 11956 (2000), provides a different three-dimensional multiple-slip generalization of
Groma's approach, retaining the densities on different slip systems as independent order parameters and incorporating the momentum field corresponding to the moving dislocations (where our theory is overdamped). El-Azab's approach has not been implemented numerically, and the question of wall formation in his approach has not been explored.

15 P. R. Dawson, D. P. Mika, and N. R. Barton, Scripta Mater. 47, 713 (2002), keep dislocation densities on multiple slip systems, where the dependence of one slip system strength depends upon the others: their simulations show misorientation distributions between finite elements which scale as do those of experimental cell walls. The relatively sharp walls in these last simulations was one of the motivations for our analytically more tractable model.

16 J. M. Rickman and J. Vinãls, Phil. Mag. A 75, 1251 (1997).

17 The simple von Mises law used in macroscopic plasticity, to illustrate our notation, would have

$$
J_{i j}^{\mathrm{vM}}=\kappa\left(\sigma_{i j}-\frac{\sigma_{k k}}{3} \delta_{i j}\right) \Theta\left[\frac{1}{2} \sigma_{p q} \sigma_{p q}-\frac{1}{3} \sigma_{p p} \sigma_{q q}-\frac{1}{3} \sigma_{\mathrm{Y}}^{2}\right]
$$

where $\Theta[\cdot]$ is the Heaviside step function. The von Mises law has dislocation flow that is independent of the existing net dislocation density: it is appropriate on macroscopic scales where the geometrically unnecessary dislocations are thought to dominate plasticity.

18 J. Pontes, D. Walgraef, and E. C. Aifantis (2005), preprint submitted to Elsevier Science.

19 S. I. Selitser and J. W. Morris, Jr., Acta Metall. Mater. 42, 3985 (1994).

20 Darcy Hughes, private communication.

21 S. Limkumnerd and J. P. Sethna, in preparation.

22 T. Mura, Micromechanics of Defects in Solids (Kluwer Academic Publishers, Dordrecht, 1991), 2nd ed.

23 C. W. Shu and S. Osher, Jour. Comp. Phys. 83, 32 (1989).

24 W. Press, S. A. Teukolsky, W. T. Vetterling, and B. P. Flannery, Numerical Recipes in $\mathrm{C}++$ (Cambridge University Press, Cambridge, 2002), 2nd ed.

25 T. Richeton, J. Weiss, and F. Louchet, Nat. Mater. 4, 465 (2005). 
26 M. C. Miguel, A. Vespignani, M. Zaiser, and S. Zapperi, Phys. Rev. Lett. 89, 165501 (2002).

27 W. T. Read, Dislocation in Crystals (McGraw-Hill Book Company, Inc., New York, 1953).

28 D. A. Hughes, D. C. Chrzan, Q. Liu, and N. Hansen, Phys. Rev. Lett. 81, 4664 (1998).

29 J. P. Sethna, V. R. Coffman, and E. Demler, Phys. Rev.
B 67, 184107 (2003).

30 D. P. Mika and P. R. Dawson, Acta Mater. 47, 1355 (1999).

31 D. Kuhlmann-Wilsdorf, Mat. Sci. Eng. A 113, 1 (1989).

32 D. Kuhlmann-Wilsdorf, Phys. Status Solidi A 149, 225 (1995). 